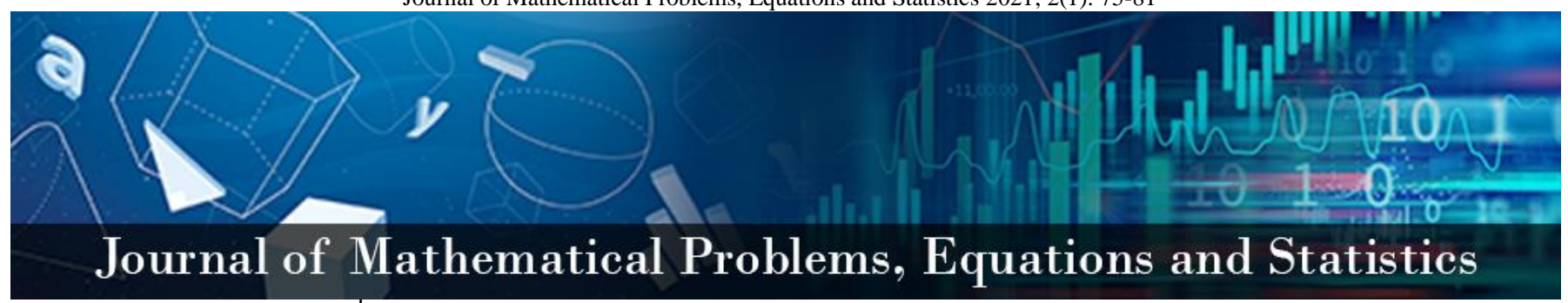

E-ISSN: 2709-9407 P-ISSN: 2709-9393 JMPES 2021; 2(1): 75-81 (C) 2021 JMPES

www.mathematicaljournal.com Received: 12-01-2021 Accepted: 14-03-2021

Ahmad Lazwardi Researcher, Universitas Muhammadiyah Banjarmasin, South Kalimantan, Indonesia

Correspondence

Ahmad Lazwardi

Researcher, Universitas

Muhammadiyah Banjarmasin

South Kalimantan, Indonesia

\section{Ops transformation}

\section{Ahmad Lazwardi}

DOI: https://doi.org/10.22271/math.2021.v2.i1a.37

Abstract

Ordinary power series have important role in discrette mathematics, recently involving complicated calculations and manipulations. The more variables included, the more complicated it will be. In this article, we will construct new perspective of ordinary power series through generating new transformation which will ignore sigma notation and minimize involving too many variables when we do some complicated calculations. The result of this research is new form of ordinary power series called Ops transformation and its basic properties.

Keywords: Ops transformation, ordinanry power series, ordinary generating function

\section{Introduction}

Generating functions are one of the most surprising and useful inventions in discrete mathematics (Meyer, 2005). Generating functions play important roles in mathematics such as solving the recurrence relations. Solving some kinds of differential equations, counting on convergence value of series, simplifying a function form and so on.

Actually counting and manipulating generating functions is not that easy. The more complicated a problem it will be the harder to manipulate. There still a lack theory discovering the method of counting the ordinary power series. There are many variables and indexes involved in general form of a power series. The simplest form of power series is the form

$$
P(x)=\sum_{n=0}^{\infty} a_{n}(x-c)^{n}
$$

The above equation contains several variables involving such as $x, n, a_{n}$, and $c$. For more complicated form will take the form

$$
P(x)=\sum_{n=0}^{\infty} a_{n}(\gamma(x)-c)^{n}
$$

Which is replacing free variable $x$ by some smooth function $\gamma$.

There are several ways to simplify the methods to solve such equations. One of them is by transforming such equations to the other isomorphics spaces which is simpler to analyze it. Wilf (1994) stated the notion of power series as following.

Definition 1.1. Let be a sequence of real numbers. A formal power series is an expression of form

$$
a_{0}+a_{1} x+a_{2} x^{2}+\ldots
$$

Furthermore, the sequence is called coefficient sequence of the corresponding expression (1.3). It's trivial to check that the collections of power series is a real linear space which is isomorphic to the real sequence space by mapping 


$$
\left\{a_{n}\right\} \mapsto \sum_{n=0}^{\infty} a_{n} x^{n}
$$

One of the benefit of power series is to solve the recurrence relation. As an example lets take a look at Fibonacci equation

$$
F_{n+2}=F_{n}+F_{n+1}\left(n \geq 1, F_{0}=0, F_{1}=1\right)
$$

Wilf (1994) solved it by modelling the relation to a generating function

$$
F(x)=\sum_{n=0}^{\infty} F_{n} x^{n}
$$

Multiplying (1.5) by $x$ one can get the left side

$$
F_{2} x+F_{3} x^{2}+F_{4} x^{3}+\ldots=\frac{F(x)-x}{x}
$$

and the right side will be

$$
\left\{F_{1} x+F_{2} x^{2}+\ldots\right\}+\left\{F_{0} x+F_{1} x^{2}+\ldots\right\}=F(x)+x F(x)
$$

Hence the equation become

$$
\frac{F(x)-x}{x}=F(x)+x F(x)
$$

Therefore we conclude

$$
F(x)=\frac{x}{1-x-x^{2}}
$$

Now we have to transform back $F(x)$ to its original form to find out the value of coefficient $F_{n}$ corresponding to McLaurin

series of $\frac{x}{1-x-x^{2}}$.

By some algebra treatment one can get

$$
\begin{aligned}
& \frac{x}{1-x-x^{2}}=\frac{x}{\left(1-x r_{+}\right)\left(1-x r_{-}\right)} \\
& =\frac{1}{\left(r_{+}-r_{-}\right)}\left(\frac{1}{\left(1-x r_{+}\right)}-\frac{1}{\left(1-x r_{-}\right)}\right) \\
& =\frac{1}{\sqrt{5}}\left(\sum_{n=0}^{\infty} r_{+}^{n} x^{n}-\sum_{n=0}^{\infty} r_{-}^{n} x^{n}\right) \\
& =\sum_{n=0}^{\infty} \frac{1}{\sqrt{5}}\left(r_{+}^{n}-r_{-}^{n}\right) x^{n} \\
& =\sum_{n=0}^{\infty} F_{n} x^{n}
\end{aligned}
$$


by $r_{ \pm}=(1 \pm \sqrt{5}) / 2$.

Therfore we get the solution $F_{n}=\frac{1}{\sqrt{5}}\left(r_{+}^{n}-r_{-}^{n}\right), n=0,1,2, \ldots$

Thats one fo the most popular example hoow do we apply ordinary power series a.k.a generating functions to solve recurrence relation problems.

Note that manupulating some generating functions is not always trivial expecially when we have to solve teh series which is not in standard form.

Here is an example how does ordinary power series takes form which is not in standar form

$F(x)=\sum_{n=2}^{\infty} \frac{(n+1)^{2}}{(n+1) !} x^{4 n}$

The above expressiosn is not in standard form of generating function due to $n$ is begun from 2 and $x$ is powered by $4 n$.

So this is another gab of generating function theory that will becom state of art of this article topics.

Wilf (1994) has an idea to assign the power series to some analytics function by the following definition.

Definition 1.2. The symbol $f \stackrel{O_{p s}}{\longleftrightarrow}\left\{a_{n}\right\}$ means the series $f$ is the ordinary power series (Ops) generating function for sequence $\left\{a_{n}\right\}$.

The above definition results several properties as following.

Suppose $f \stackrel{O p s}{\longleftrightarrow}\left\{a_{n}\right\}$, then what generates $\left\{a_{n+1}\right\}$ ?

By little work we will get

$\sum_{n=1}^{\infty} a_{n+1} x^{n}=\frac{1}{x} \sum_{m=1}^{\infty} a_{m+1} x^{m}=\frac{f(x)-f(0)}{x}$

Therefore we have

$\left(\frac{f-a_{0}}{x}\right) \stackrel{O p s}{\longleftrightarrow}\left\{a_{n+1}\right\}$

Inductively we get for some positive integer $h$.

$\left(\frac{f-a_{0}-\ldots-a_{n-1} x^{n-1}}{x^{n}}\right) \stackrel{O p s}{\longleftrightarrow}\left\{a_{n+h}\right\}$

The last expression is not simple enough and thus not so efficient to solve many generating function with complicated forms. We will find more efficient idea to simplify the expression through this topic.

\section{Material and Methods}

In this article, we will construct the definition of Ops transformation and make sure that the definition should be well defined. After that we will analyze some of its basic properties. Next we will try to apply Ops transformation and its properties to solve some ordinary power series calculations.

\section{Result and Discussion}

If we observe at (1.10) the sigma notation and variable $x$ dont play so important role which we can ignore that for a while. So in order to do some fast, effective calculations sometime we might to eliminate $x$ and sigma notation for a while.

Observe the following definition. Recall that

$\mathbb{R}^{\mathbb{N}}=\left\{\left\{a_{n}\right\}: a_{n} \in \mathbb{R}\right\}$

i.e the space of all real sequences. Its trivially one can show that $\mathbb{R}^{\mathbb{N}}$ is a linear space over $\mathbb{R}^{\mathbb{R}}$. Now we define a transformation as following 
Definition 3.1. Ops transformation is mapping

\section{Ops: $\mathbb{R}^{\mathbb{N}} \times \mathbb{R} \rightarrow \mathbb{R}^{*}$}

by

$$
\operatorname{Ops}\left(\left\{a_{n}\right\}\right)(x)=\sum_{n=0}^{\infty} a_{n} x^{n}
$$

Its well defined because arbitrary real power series has radius of convergence. Observe sometime we can write just $\operatorname{Ops}\left(\left\{a_{n}\right\}\right)$ instead of $\operatorname{Ops}\left(\left\{a_{n}\right\}\right)(x)$. We can assume $\operatorname{Ops}\left(\left\{a_{n}\right\}\right)$ as a $C^{\omega}(D)$ function (i.e real analytics function) from some $\operatorname{disk} D \subset \mathbb{R}$ determined by its radius convergence.

Another expression of ops transformation is to write it implicitly by unspecified notation as McBride(1971). Its written as

$$
G\left(a_{n} ; x\right)=\sum_{n=0}^{\infty} a_{n} x^{n}
$$

Its fine, but we will always involve variable $x$ in every calculation, which means the expression (3.12) still more effecient in some situations.

Now we will explore some of Ops Transformations properties

Theorem 3.2. For each $\left\{a_{n}\right\} \in \mathbb{R}^{\mathbb{N}}, \operatorname{Ops}\left(\left\{0, a_{0}, a_{1}, \ldots\right\}\right)=x O p s\left(\left\{a_{n}\right\}\right)$

Proof: For each $x$ we have

$$
\begin{aligned}
& x \operatorname{Ops}\left(\left\{a_{n}\right\}\right)(x)=x \sum_{n=0}^{\infty} a_{n} x^{n} \\
& =a_{0} x+a_{1} x^{2}+\ldots \\
& =0+a_{0} x+a_{1} x^{2}+\ldots \\
& =\operatorname{Ops}\left(\left\{0, a_{0}, a_{1}, \ldots\right\}\right)
\end{aligned}
$$

Inductively theorem 3.2 gives concequency as follow

Corollary 3.3. For each $\left\{a_{n}\right\} \in \mathbb{R}^{\mathbb{N}}$, Ops $\left(\{\underbrace{0,0, \ldots, 0}_{k-\text { terms }} a_{0}, a_{1}, \ldots\}\right)=x^{k} \operatorname{Ops}\left(\left\{a_{n}\right\}\right)$

As well as Benderier(2002) explains generating function, we have following properties

Theorem 3.4. For each $\left\{a_{n}\right\} \in \mathbb{R}^{\mathbb{N}}, O p s\left(\left\{a_{n}\right\}\right)-a_{0}=x O p s\left(\left\{a_{n+1}\right\}\right)$.

Proof: For each $x$ lies on the domain we have

$$
\begin{aligned}
& \operatorname{ops}\left(\left\{a_{n}\right\}\right)(x)-a_{0}=\sum_{n=1}^{\infty} a_{n} x^{n} \\
& =\sum_{n=1}^{\infty} a_{n+1} x^{n+1} \\
& =x \sum_{n=1}^{\infty} a_{n+1} x^{n}
\end{aligned}
$$


$=x O p s\left(\left\{a_{n+1}\right\}\right)(x)$.

Thus inductively we get

Corollary 3.5. For each $\left\{a_{n}\right\} \in \mathbb{R}^{\mathbb{N}}$, Ops $\left(\left\{a_{n}\right\}\right)-\sum_{n=0}^{k-1} a_{n} x^{n}=x^{k} \operatorname{Ops}\left(\left\{a_{n+k}\right\}\right)$

As another consequancy we also have

Corollary 3.6. For each $\left\{a_{n}\right\} \in \mathbb{R}^{\mathbb{N}}$, Ops $\left(\left\{a_{n+k}\right\}\right)=\frac{\operatorname{Ops}\left(\left\{a_{n}\right\}\right)-\sum_{n=0}^{k-1} a_{n} x^{n}}{x^{k}}, x \neq 0$

Ops transformation is failed to be linear transformation from $\mathbb{R}^{\mathbb{N}} \times \mathbb{R}$ to $\mathbb{R}^{*}$ because $O p s\left(\left\{a_{n}\right\}\right)(x+y)$ is not necessary equals to $o p s\left(\left\{a_{n}\right\}\right)(x)+O p s\left(\left\{a_{n}\right\}\right)(y)$. But if we set $x$ to be fixed then we have

$O p s\left(\left\{a_{n}\right\}+\left\{b_{n}\right\}\right)(x)=O p s\left(\left\{a_{n}\right\}\right)(x)+O p s\left(\left\{b_{n}\right\}\right)(x)$

By ignoring $x$ for a while we can write

$O p s\left(\left\{a_{n}\right\}+\left\{b_{n}\right\}\right)=O p s\left(\left\{a_{n}\right\}\right)(x)+O p s\left(\left\{b_{n}\right\}\right)$

Its easy to check that for each $\alpha \in \mathbb{R}$

$O p s\left(\alpha\left\{a_{n}\right\}\right)=\alpha O p s\left(\left\{a_{n}\right\}\right)$

Now we shall observe what value of Ops transformation for the product of two power series. Suppose we have

$$
\begin{aligned}
& \operatorname{Ops}\left(\left\{a_{n}\right\}\right)(x)=\sum_{n=0}^{\infty} a_{n} x^{n} \text { and } \operatorname{Ops}\left(\left\{b_{n}\right\}\right)(x)=\sum_{n=0}^{\infty} b_{n} x^{n} \text { then } \\
& \operatorname{Ops}\left(\left\{a_{n}\right\}\right) \operatorname{Ops}\left(\left\{b_{n}\right\}\right)(x)=\left(\sum_{n=0}^{\infty} a_{n} x^{n}\right)\left(\sum_{n=0}^{\infty} b_{n} x^{n}\right) \\
& =\left(\sum_{n=0}^{\infty}\left(\sum_{k=0}^{n} a_{k} b_{n-k}\right) x^{n}\right) \\
& =\operatorname{Ops}\left(\sum_{k=0}^{n} a_{k} b_{n-k}\right)(x)
\end{aligned}
$$

This will result the following theorem

Theorem 3.7. For each $\left\{a_{n}\right\}\left\{b_{n}\right\} \in \mathbb{R}^{\mathbb{N}}$, Ops $\left(\left\{a_{n}\right\}\right) \operatorname{Ops}\left(\left\{b_{n}\right\}\right)=\operatorname{Ops}\left(\sum_{k=0}^{n} a_{k} b_{n-k}\right)$.

Next we will observe for the derivative of Ops transformation. As we know that any power series are smooth functions, so we are always able to define the derivative of Ops transformation with respect to $x$.

Now lets observe the following definition

Definition 3.8. Let $\left\{a_{n}\right\} \in \mathbb{R}^{\mathbb{N}}$, and $c$ be an interior point of domain of $o p s\left(\left\{a_{n}\right\}\right): \mathbb{R} \rightarrow \mathbb{R}$. Define

$$
D_{x} \operatorname{Ops}\left(\left\{a_{n}\right\}\right)(c)=\frac{\operatorname{Ops}\left(\left\{a_{n}\right\}\right)(x)-O p s\left(\left\{a_{n}\right\}\right)(c)}{x-c}
$$

The above definition is no other than definition of derifative of function in usual sense. 
Now we shall explore more properties of Ops transformation related to its derivative.

Theorem 3.9. If $\left\{a_{n}\right\} \in \mathbb{R}^{\mathbb{N}}$, then $D_{x}$ Ops $\left(\left\{a_{n}\right\}\right)=\operatorname{Ops}\left(\left\{(n+1) a_{n+1}\right\}\right)$

For example we have $D_{x} \operatorname{Ops}(\{1\})=(\operatorname{Ops}(\{1\}))^{2}$ (K.Lando (2002)) As for consequence of the above theorem. There should be the following corollary

Corollary 3.10. If $\left\{a_{n}\right\} \in \mathbb{R}^{\mathbb{N}}$, then

$$
D_{x}^{k} O p s\left(\left\{a_{n}\right\}\right)=D_{x}\left(D_{x}\left(\ldots\left(D_{x} O p s\left(\left\{a_{n}\right\}\right)\right)\right)=O p s\left(\left\{\frac{(n+k) !}{n !} a_{n+k}\right\}\right)\right.
$$

Another useful property of Ops transformation is

Theorem 3.11. If $\left\{a_{n}\right\} \in \mathbb{R}^{\mathbb{N}}$, then $x D_{x} O p s\left(\left\{a_{n}\right\}\right)=O p s\left(\left\{n a_{n}\right\}\right)$

Proof: Lets observe

$$
\begin{aligned}
& \operatorname{Ops}\left(\left\{n a_{n}\right\}\right)=\sum_{n=0}^{\infty} n a_{n} x^{n} \\
& =x \sum_{n=1}^{\infty} n a_{n} x^{n-1} \\
& =x \sum_{n=0}^{\infty}(n+1) a_{n+1} x^{n} \\
& =x \operatorname{Ops}\left(\left\{(n+1) a_{n+1}\right\}\right) \\
& =x D_{x} \operatorname{Ops}\left(\left\{a_{n}\right\}\right)
\end{aligned}
$$

Inductively we can get the following property

Corollary 3.12. If $\left\{a_{n}\right\} \in \mathbb{R}^{\mathbb{N}}$, then $O p s\left(\left\{n^{k} a_{n}\right\}\right)=\underbrace{x D_{x}\left(x D_{x}(\ldots) x D_{x}\right.}_{k \text { times }} O p s\left(\left\{a_{n}\right\}\right)$

Here we will give some example how to apply Ops transformation to recurrence relation. We will solve the equation

$a_{n}+3 a_{n-1}-1=0, a_{0}=1$

We will rewrite the equation by shifting the index

$a_{n+1}+3 a_{n}-1=0, a_{0}=1$

First we transform the equation to Ops transformation form

$\operatorname{Ops}\left(\left\{a_{n+1}+3 a_{n}-1\right)=0\right.$

$=\operatorname{Ops}\left(\left\{a_{n+1}\right\}\right)+3 \operatorname{Ops}\left(\left\{a_{n}\right\}\right)-O p s(\{1\})$

$=0$

Therefore 
$\operatorname{Ops}\left(\left\{a_{n+1}\right\}\right)+3 \operatorname{Ops}\left(\left\{a_{n}\right\}\right)=\left(\frac{\operatorname{Ops}\left(\left\{a_{n}\right\}\right)-a_{0}}{x}\right)+3 \operatorname{Ops}\left(\left\{a_{n}\right\}\right)$

$\operatorname{Ops}\left(\left\{a_{n}\right\}\right)-a_{0}+3 x \operatorname{Ops}\left(\left\{a_{n}\right\}\right)=x \operatorname{Ops}(\{1\})$

By substituting, and calclutating the value of $a_{o}$ and $\operatorname{Ops}(\{1\})$ we have

$$
\operatorname{Ops}\left(\left\{a_{n}\right\}\right)+3 x \operatorname{Ops}\left(\left\{a_{n}\right\}\right)=\left(\frac{1}{1-x}\right)
$$

Therefore

$\operatorname{Ops}\left(\left\{a_{n}\right\}\right)=\left(\frac{1}{(1-x)(1+3 x)}\right)=\frac{1}{4(1-x)}+\frac{3}{4(1+3 x)}=\operatorname{Ops}\left(\left\{\frac{1}{4}+\frac{3}{4}(-3)^{n}\right\}\right)$

Eliminating the Ops we now have

$$
a_{n}=\left(\frac{1}{4}+\frac{3}{4}(-3)^{n}\right)
$$

which is the solution of above recurrence relation.

\section{Conclusion}

From this article, we conclude that Ops transformation is mapping

Ops: $\mathbb{R}^{\mathbb{N}} \times \mathbb{R} \rightarrow \mathbb{R}^{*}$

by

$$
\operatorname{Ops}\left(\left\{a_{n}\right\}\right)(x)=\sum_{n=0}^{\infty} a_{n} x^{n}
$$

\section{Referencess}

1. Benderier C. Generating Functions for Generating Trees 2002, 29-55.

2. Brent RP. Fast Algorithm for Manipulating Formal Power Series 1966;4:581-595.

3. Lando KS. Lectures on Generating Functions. American Mathematical Society. United States 2002.

4. Meyer RA. Generating Functions, Masachusetts Institute of Technology 2005.

5. Wilf. Generating Functionology. Academic Press, Inc. Pensylvania 1994.

6. Wikipedia. https://en.wikipedia.org/wiki/Generating_function

7. Wolfram Mathworld. https://mathworld.wolfram.com/GeneratingFunction.html 